\title{
Designing Library Instruction for Undergraduates: Combining Instructional Systems Design and Naturalistic Inquiry
}

\section{Delia Neuman}

An instructional systems design (ISD) process guided the creation of $M A J I K / 1$, a HyperCard program delivering basic, individualized library instruction in preparation for classroom teaching in more advanced topics. A technique derived from naturalistic inquiry (NI) was incorporated into the program's formative evaluation, which was administered to twenty-eight uppercIassmen at the University of Maryland, College Park. Analysis of the data revealed that students had little difficulty with the content of the program but extensive difficulty in navigating within the HyperCard environment. The combination of ISD and NI suggests an effective approach to developing similar materials for library education.

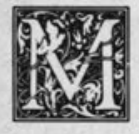

agazine and Journal Instructional Kit, Part 1 (MAJIK/1) is a HyperCard introduction to periodical indexes, to the procedures for using the University of Maryland, College Park (UMCP) Serials List to locate specific articles, and to the arrangement of periodicals throughout the UMCP Libraries System. MAJIK/1, the first and most basic of an anticipated four-module system on locating periodical articles at UMCP, is designed both to be used in conjunction with UMCP junior composition courses and to stand on its own as a self-paced module for individuals seeking to review and update their skills.

The creation of the module followed an eight-step process based on instructional systems design (ISD) as explicated by Robert Gagne and Leslie Briggs, Walter Dick and Lou Carey, Jerrold Kemp, and others. ${ }^{1}$ Derived from research and theory in the fields of learning, communication, systems engineering, and instructional technology, ISD consists of a set of principles and techniques for systematically designing and developing

Delia Neuman is assistant professor, College of Library and Information Services, University of Maryland, College Park, Maryland 20742-4345. The Council on Library Resources Cooperative Research Grant Program supported the research reported in this paper. The author wishes to thank two University of Maryland, College Park (UMCP) staff for their contributions to the project and their suggestions for this article: Rebecca Jackson, UMCP Libraries Coordinator for Instructional Services and coprincipal investigator for the project, and Sandra Dwiggins, doctoral student in the College of Library and Information Services. 
effective instruction. The process is generally defined in terms of the specific steps designers follow to create a product. For MAJIK/1, these steps can be divided into two segments. The first six-needs analysis, learner analysis, specification of goals and objectives, development of test items, selection of the delivery medium, and development of materials and activities - comprised the design phase. The final two steps-formative evaluation and revision-comprised the evaluation phase.

While several authors have suggested an ISD approach to designing bibliographic instruction, none has sought to incorporate techniques of naturalistic inquiry (NI) into that approach. ${ }^{2} \mathrm{NI}$, a research paradigm gaining renewed prominence in all areas of social science inquiry, is generally defined by contrasting it with the so-called rationalistic paradigm that guides more traditional social science research. Thus, NI is characterized by five major differences from the rationalistic paradigm: its focus on "natural" rather than experimental settings, its attempt to generate contextbound insights rather than universal generalizations, its assumption that the researcher rather than any paper-andpencil or electronic tool is the preferred instrument for data collection and analysis, its emphasis on issues of importance to those who participate in the research rather than on questions posed a priori by the researcher, and its reliance on the collection and analysis of verbal rather than quantitative data as the primary source of its conclusions. In MAJIK/1, a particular NI techniquederived by the researcher from the larger NI arsenal and defined by her as "interactive observation" - was the primary strategy used in the formative evaluation.

This paper describes the MAJIK/1 project in an attempt to demonstrate the potential effectiveness of combining ISD and NI in the design of library instruction materials for undergraduates. Complete details of the effort appear in the final project report, which is available upon request. ${ }^{3}$ This paper has two pri- mary foci: first, it highlights the most notable illustrations of the use of ISD in the project and, second, it concentrates on the contribution of the interactive-observation strategy to the project's formative evaluation phase.

\section{DESIGN OF MAJIK/1}

\section{Step 1: Needs Analysis}

Needs analysis, the attempt to identify the gap between actual and desired learner performance, is designed to ensure that ISD projects are undertaken to meet genuine instructional needs that are clearly understood and thoroughly defined. The experience of designing MAJIK/1 underscores the significance of this critical but often neglected step because the actual instructional need was not apparent at the beginning of the project but revealed itself as the process evolved.

The UMCP Library Instruction Program for undergraduates is comprehensive but consists primarily of two one-hour instructional sessions. The first, an introduction to basic library resources and their uses, is offered in conjunction with the freshman composition program. The second, an explication of more advanced library research skills, is offered in conjunction with the junior composition program. Problems identified by UMCP Libraries staff responsible for the second session suggested the general need for the module: these staff consistently encountered students who were not ready to learn the higher-level concepts included in the junior curriculum. Many of the students had not mastered the basic concepts covered in the freshman component. The staff was therefore forced to spend its limited instructional time covering these basics rather than addressing such topics as the formulation of successful search strategies. In order to address this problem, the UMCP Libraries System Associate Director for Public Services convened the UMCP Library Education Committee in the spring of $1988 .{ }^{4}$

Charged originally with improving the junior component of the Library In- 
struction Program, the committee initially focused on the instructors' experiences in that setting. Discussion, however, soon began to focus on improving the freshman component. Committee members reasoned that a point of achievement at this level is a prerequisite to successful instruction for juniors. Ultimately, however, the committee recognized that the key instructional problem did not necessarily involve either the freshman or the junior component. Transfer students and students who place out of the freshman composition requirement do not necessarily encounter the basic concepts covered in the freshman component; other students do not necessarily use those concepts until two years after instruction. Therefore, many juniors' lack of appropriate background is clearly extraneous to the quality of the freshman component. The committee concluded that the primary need was not a revision of the freshman or the junior program but the development of an intermediate instructional experience for any students who had to master basic concepts before they could profit from advanced instruction.

\section{Step 2: Learner Analysis}

Unlike the needs analysis phase of the project, the learner analysis for MAJIK/1 was straightforward. The committee identified the general audience as juniors taking the UMCP's required upper-level composition course, which includes a requirement for a paper involving library research. More specifically, the audience would be transfer students, students who had placed out of the required freshman composition course, and other students who wanted to refresh their skills.

This broad range of students precluded any finer delineation of learner characteristics. Although students were sure to vary widely in the important characteristics of age, prior learning experiences, special abilities and disabilities, and specific entry behaviors, the nature and extent of such variations within a large group were both unknowable and beyond the scope of a single package to accommodate. Consequently, the materials were developed at a general level to make them accessible to a broad audience.

\section{Step 3: Specification of Goals and Objectives}

Step 3 was similarly straightforward, consisting of committee members identifying the concepts students should master before encountering advanced instruction. Two committee members who became the primary members of the instructional design team for MAJIK/1 translated these concepts into the goals and objectives that provided the framework for the remainder of the project. As shown in appendix A, four major categories of goals and objectives defined the four major components of the final materials: introduction to periodical indexes, using periodical indexes, using the UMCP Serials List, and locating periodicals in UMCP Libraries. For the purposes of this paper, the objectives have been selected and condensed from their original, more UMCP-specific form in order to highlight their scope and detail.

\section{Step 4: Development of Test Items}

Thirty-five test items based on the objectives for MAJIK/1 were devised for the formative evaluation of the materials. These items were compiled into simple pre- and posttests that were administered to students in an attempt to determine the contributions of the program to their learning.

\section{Step 5: Selection of the Delivery Medium}

Several considerations merged to suggest HyperCard as the most appropriate delivery mode for MAJIK/1. First, because not all students need elementary instruction, an independent, standalone module seemed the best solution to the instructional problem. Although other independent learning formats were considered (for example, videocassette), computer-assisted instruction (CAI) was ultimately chosen because of its individualization, self-pacing, immediate feedback, and flexible scheduling. 
CAI enables individual students to progress through the presentation of concepts and the completion of practice exercises at their own rates rather than at the pace dictated by large-group instruction. Moreover, well-designed CAI allows students to make choices about the sequence they will follow and, in some instances, the concepts they will study rather than requiring them to proceed according to the needs of a larger group. The immmediate feedback provided for individual answers avoids misleading students and allows them to monitor the success of their own learning. Finally, because CAI can occur at any time the hardware is available-for example, in an all-night computer labstudents can arrange their own instructional time without regard to the constraints imposed by class schedules. For all these reasons, the committee decided to make MAJIK/1 a CAI module available in several campus locations, including a lab in the UMCP Undergraduate Library. Students could be assigned to complete the package independently before the library instruction session, freeing instructors to use their limited class time to focus on more advanced library research topics.

\section{Computer-assisted instruction}

(CAI) was ultimately chosen because of its individualization, self-pacing, immediate feedback, and flexible scheduling.

HyperCard is the innovative software that allows Macintosh computers to present CAI (and other kinds of programs) in a nonlinear, associational form rather than in the hierarchical structure inherent in traditional software. Because users move through HyperCard programs directly from one segment to another rather than through a series of segments linked in a hierarchy, HyperCard offers especially rapid and flexible movement through information according to individual preferences and needs. HyperCard was chosen for deliv- ery of MAJIK/l in order to exploit this instructional potential, to provide appropriate software for new Macintoshes that had recently been purchased by the UMCP Libraries, and to allow the designers to explore the possibilities of this exciting new format as they relate to library instruction.

Students could be assigned to complete the package independently... freeing the instructors to use their limited class time to focus on more advanced library research topics.

HyperCard programs are called "stacks," and individual screens are called "cards." Users move through stacks by using the Apple mouse to move the cursor to a particular area of a card called a "button" and depressing the panel on top of the mouse to "click on," or select, that button; the program then moves directly to the program segment indicated by the button. A button can be a special typeface (such bold) or an icon, a visual symbol of a program segment. In MAJIK $/ 1$, for example, the question mark icon is the button for the "help" segment. Students can click on this button to move to the card that reviews general concepts important to the program.

Figures 1 through 4 show how MAJIK/1 takes advantage of HyperCard through a structure that allows flexibility of movement across and within components. Figure 1, the table of contents card, shows that students may choose to go through one, two, three, or all four segments of the package in any order. Students may also proceed in a linear fashion by clicking on the "next" and "back" buttons to move forward and backward through the cards in sequence. Figure 2, which contains all the major icons used in the program, shows a variety of options. At almost any point in MAJIK/1, students may leave one segment and go to another by clicking on the icon for the desired destination. Students may also click on the " 1 " icon to 


\title{
$\stackrel{\text { tonrins }}{=}$ Tabile of Gontents
}

(Click on the boldface word and you will go to that section).

\author{
Introduction
}

Periodical Inderes

UMCP Serials List

Where to Find Periodicals

Figure 1. Table of Contents

You have finished the Introduction.

itg Remember --

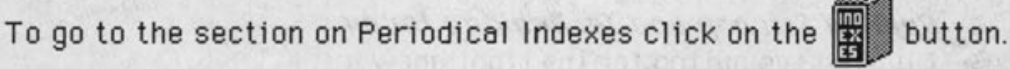

To go to the section on the UMCP Serials List click on the

To go to the section on where to Find Periodicals click on the

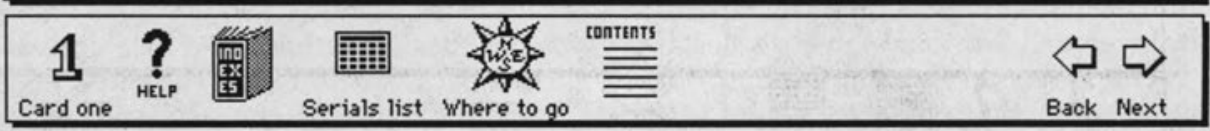

Figure 2. Major MAJIK/1 Icons 
If you are just beginning to use periodical articles for research, start with the section called Periodical Indexes.

You will learn how to:

-choose a periodical inder,

-choose appropriate subject headings,

-identify the items within each citation,

-find periodical titles on the UMCP Serials List,

- locate periodicals within the UMCP Libraries System.

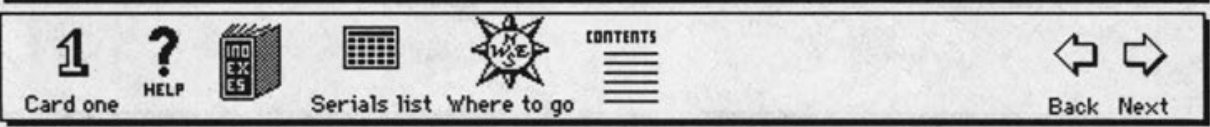

Figure 3. Boldface for Definitions

5. Safety in engineering design and construction

a. Nationa/ Neuspaper /ndes

b. Art Indes

c. Applied Science and Technalogy Indes

(9) Click on the button for the right answer.

6. Microcomputer applications in business

a. Business Periadicals /ndes

b. Applied Science and Technalagy /ndes

c. Aeader's Guide to Periadical Literature

(9) Click on the button for the right answer.

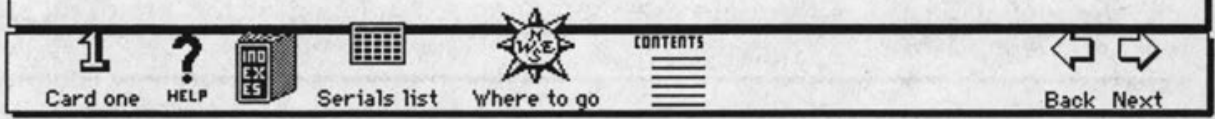

Figure 4. Buttons for Checking Answers 
return to the title card, the "help" icon to review general program information, or the "contents" icon to return to the table of contents card. This card serves as an orientation point for students, a familiar home base to which they can return at any time to reenter any component of the program. Figure 3 shows that, within components, students may click on boldface terms and phrases to move to definitions of key concepts according to their own individual needs for information. Figure 4 shows that they may click on the icon of the button to check their answers in the practice exercises. Thus MAJIK/1 provides a variety of options for movement within and across segments. This structure capitalizes on the advantages of CAI in general and HyperCard in particular to enable students to determine their own paths through the material and to tailor the time spent with the program to best meet their own needs.

\section{Step 6: Development of \\ Materials and Activities}

The majority of the MAJIK/1 development time involved creating the prototype HyperCard materials. While this is typical of any ISD project, the composition of the MAJIK/1 design team led to an interplay that is not typical of ISD projects but that was a strength of this one. The subject matter expert (the UMCP Libraries Coordinator for Instructional Services) was experienced in instructional techniques as well as content; the instructional designer (a faculty member of the UMCP College of Library and Information Services) was knowledgeable about library issues as well as about instruction; and the technical support specialist (a programmer who was also completing an M.L.S.) brought content knowledge as well as programming expertise to this role. Although project tasks were divided according to the traditional functions of each of these roles, the particular backgrounds of the team members enhanced their collaboration throughout the development of MAJIK/1.
This development occurred in four stages, each corresponding to one of the four content areas covered by the materials: introduction to periodical indexes, using periodical indexes, using the UMCP Serials List, and locating periodicals in UMCP Libraries. Each component includes the presentation of the appropriate content and instructions for navigating in a HyperCard environment. The two middle components, which comprise the major portion of the instruction, include exercises in which students actually use the concepts presented. The total package is comprised of approximately 230 cards: eighty containing primary instructional information and 150 containing definitions, answers, and similar supplementary material.

\section{EVALUATION OF MAJIK/1}

\section{Step 7: Formative Evaluation}

During the summer of 1989, three composition classes at UMCP participated in the formative evaluation of MAJIK/1. One class served as a pilot for the evaluation processes and instrumentation, while the other two furnished the data upon which revision decisions were made. Twenty-four students provided pre- and posttest data; twenty-eight provided naturalistic data through the interactive observations that were the heart of the evaluation strategy.

\section{Data Collection}

After hearing an explanation of the project and completing the pretest, participating students used the materials while a doctoral student from the College of Library and Information Services conducted the interactive observations. Upon completion of the program, students took the posttest.

The pretest-posttest strategy is typical of formative evaluation approaches, while the interactive-observation strategy is not. Derived from the principles and procedures of naturalistic inquiry (NI) as described by Egon Guba, Yvonna Lincoln and Egon Guba, and Delia Neu- 
man, the strategy requires the researcher to interact freely with the students as they use the prototype version of instructional materials. ${ }^{5}$ For this project, the strategy involved three tactics: interrupting students as they used the package to ask about problems they were encountering; questioning them upon completion of each component of the program; and interviewing them upon completion of the entire experience to capture their insights about strengths, weaknesses, and necessary and desirable improvements.

The observer had been trained specifically for this project and used a protocol designed to structure both her observations and the planned analysis of the data. The two-page protocol contained six sections: an identification section for the student's name and the date and time of the observation; a summary section for the student's summary comments; and one section for each of the four major content areas of the program. Items within those sections addressed whether the section had been selected; what strengths and weaknesses the student had found in the section's presentation of concepts and tasks; what strategies the student had used to complete the section; what comments and suggestions for revision the student could offer; and what comments and suggestions the observer could make based on the observations.

The use of NI data collection strategies in a contrived setting like an ISD evaluation is inconsistent with one of the basic tenets of $\mathrm{NI}$-that the paradigm is best used to investigate phenomena that occur in natural settings. Using interactive observations capitalizes, however, on several other NI tenets-that individuals' perceptions and experiences are the most important data and that interaction between the researcher and the participant yields the best understanding of those perceptions and experiences. Incorporating naturalistic strategies into formative evaluation thus enables the instructional designer to get maximum revision information from learners.
In the case of MAJIK/1, the approach produced the most important information derived from the evaluation: that students had more difficulty navigating the package than they had mastering its content. The design team had not anticipated this difficulty, and, consequently, did not include it in the categories in the observation protocol. Nevertheless, the observations revealed that navigation was the most important area to be addressed during the program's revision. If the evaluation had relied only on the pre- and posttests, which addressed the program's content, the most serious flaw in the materials, which related to the processes required to traverse that content, might have been overlooked.

\section{Data Analysis}

Data analysis proceeded along two fronts: statistical treatment of the preand posttest scores and naturalistic assessment of the observational data. As is appropriate in formative evaluation, the evaluation design involved neither randomization nor a control group, and the statistical analysis attempted only to draw general inferences about the instructional effectiveness of the materials for the participating students. ${ }^{6}$ The analysis of the basic differences between preand posttest scores for the twenty-four students for whom such data were available revealed a modest gain, an increase of approximately 15 percent in mean scores, suggesting that $\mathrm{MAJIK} / 1$ has promising instructional value.

Because of the small number of students (28) observed using the material, the observational data were analyzed manually rather than by computer. Preliminary data analysis consisted of creating tables that displayed the data for each student according to each of the categories in the observation protocol. Final analysis involved summarizing this information by category (including the new "HyperCard" category revealed during the observations) and drawing the conclusions and implications described below. In contrast to the encouraging but cursory result provided by the pretest-posttest analysis, analysis of the 
observational data yielded a number of specific and incisive suggestions for revising MAJIK/1.

Most significantly, the analysis revealed the details of students' difficulty in navigating the program. Initially, for example, several students were unfamiliar with the standard HyperCard notions of clicking, buttons, and icons and had to be instructed by the observer. A quarter of the students expressed dismay about the amount of navigational material-particularly icons-they were expected to know in order to use the program: "We're supposed to remember all these?" A number of students also failed to remember that clicking on a boldface item would lead to its definition, and several made navigational errors due to their unfamiliarity with moving through HyperCard material: "How do you get back to the...[e.g. the help] section?"

Other findings emerged from the analysis of the observational data as well. Many students were pleased with the MAJIK/1 practice exercises. Students gave evidence of active engagement (e.g., nodding their heads as they proceeded) and offered such positive comments as "good," "helpful," and "There were enough exercises to make you feel comfortable with the material." The number and tenor of students' comments suggest that the exercises are a useful and attractive feature. The presence of both behavioral and verbal data engenders more confidence in that finding than would be warranted by similar survey or questionnaire data.

However, several students found the directions for completing the exercises unclear, indicating that these should be revised. The primary source of students' confusion seems to be the design of the exercises rather than the directions themselves. The exercises are a series of multiple-choice questions with lettered options and an instruction to answer each question mentally and then click on a button to confirm the correctness of the answer. Almost half the students, however, initially tried to enter the letters of their choices, expecting the program to accept and judge their responses in the now-familiar format of comparable CAI tasks. Apparently, because the MAJIK/1 task ran counter to their expectations, it initially disconcerted the students. They overcame their confusion readily, however, suggesting that the problem is a minor one.

Almost all students' summary comments about MAJIK/1 were positive. Students focused on such features as the program's good graphics (13 comments), clear text (10), helpful practice exercises (9), self-pacing (7), and ease of movement within the package (5). Students' general comments focused on the informative, useful, easy-to-use, and creative nature of the materials, with one student commenting on the good balance between text and graphics and another pronouncing the program "very worthwhile." One student noted that "[I] learned as much from the module as I had in class."

Nine students in the group explained that the materials had been a review for them, while the others did not offer that information. Several suggested that the package would be good for freshmen, sophomores, and transfer students. One recommended that it be compulsory for freshmen and another wished it had been available "when I was a freshman."

Not all summary comments were positive: five students criticized the amount of detail in the program, five complained that the icons are difficult to remember, three protested the amount of time it took to complete the package, and two noted confusion over the use of boldface. And as one candid critic noted, "The only reason a person would go through all this would be if the professor made them."

In summary, both statistical and naturalistic analyses of student performance suggest that the prototype package effectively met the needs it set out to address. In particular, students' comments and behaviors indicated that the limited number of students who used the materials found them attractive, useful, and reinforcing. More importantly for this project, the level of detail provided by 
the analysis of the observational data ensured that the MAJIK/1 revisions directly and thoroughly addressed the most problematic aspects of the package.

\section{Step 8: Revision}

Drawing upon this analysis, the design team incorporated a number of revisions to improve the package's navigability for all students - even those unfamiliar with HyperCard. Brief labels were added to the icons, enhancing their visual stimulus with a written one. Several icons were made more representative of the functions they symbolize. Hints and prompts about the meanings and uses of the various buttons were incorporated directly into appropriate screens to serve as reminders throughout the package.

Most importantly, an optional tutorial explaining the features of the program and suggesting navigational strategies was included at the beginning. The tutorial includes instruction in the meanings and uses of buttons and both graphic and verbal information designed to enhance students' abilities to proceed independently. An overview of the entire package as well as overviews of individual components were included to help students develop an appropriate conceptual structure to guide their progress through the materials. Similarly, instructions in such navigational strategies as using the table of contents as a focal point were included to give students a way to orient themselves throughout the program.

\section{CONCLUSIONS}

The MAJIK/1 project has resulted in several products of use to library instructors: the program itself, increased knowledge about the application of HyperCard to library education, and a development approach that is potentially the most significant outcome of the project. This ISD/NI approach, combining insights from two separate academic disciplines, provides guidance for the entire development cycle for library education materials. Following this sys- tematic and straightforward approach can result in materials that effectively meet the needs of the students for whom they are designed.

The MAJIK/1 project has resulted in several products of use to library instructors... [including] a development approach that is potentially the most significant outcome of the project.

In the design phase, the use of ISD techniques provided well-grounded analyses of instructional needs and learner characteristics, a precise specification of relevant goals and objectives, a writing of test items directly related to those goals and objectives, and a systematic development of prototype materials designed to address these goals and objectives for the identified learners. Moreover, the "team" approach common to ISD efforts led to the collaboration of experts in three areas-library education, instructional message design, and HyperCard programming - to ensure that all appropriate areas of expertise were tapped. The iterative process characteristic of ISD guaranteed that all three developers would review and comment upon one another's efforts, ensuring that the prototype reflected the insights of the group as a whole.

Formative evaluation and prototype revision are inherent in ISD, but formally and extensively employing NI techniques during evaluation in order to get optimal information for revision is a new strategy. In this project, however, drawing upon the assumptions and techniques of NI brought important strengths to the MAJIK $/ 1$ materials. Of obvious value are the insights related to specific revisions gleaned through the interactive observations. Even more significant, however, is the discovery of the major deficiency in the original program, the lack of adequate support for navigating the HyperCard materials successfully. Thus, while the more traditional pretest-posttest approach yielded 
little information for revision (and none on this important topic), NI provided insight into the revisions that are most likely to enhance the quality and utility of the materials. This insight suggests an area of concern for the designers of other instructional materials in a hypertext format as well: student groups will undoubtedly include learners who are unfamiliar with the terminology, conceptual structure, and navigational possibilities inherent in such a format. Therefore, designers must incorporate features that will enable all students to use hypertext packages independently.

The hallmark of NI is its underlying assumption that the individuals in a research study are participants with valuable insights to offer rather than subjects to be tested according to the researcher's own preconceptions and beliefs. The NI strategy used in this study capitalized on this assumption and ensured that repre- sentative individuals had an opportunity to react to the materials, to identify effective and ineffective instructional approaches within them, and to suggest ways to improve the product. Clearly, incorporating this information into the ISD revision step leads to a more suitable product.

The creation of MAJIK/l thus provided a test case for combining the techniques of ISD and NI into a new approach for developing and testing an interactive instructional package for basic library education. The MAJIK/1 experience suggests that the basic approach is feasible for similar development projects. And because this project has served as a preliminary validation of the approach, it can be used in similar projects with confidence that the resulting materials will be effective with and attractive to the audiences for whom they are designed.

\section{REFERENCES AND NOTES}

1. Robert M. Gagne and Leslie J. Briggs, Principles of Instructional Design, 2nd ed. (New York: HoIt, 1979), passim; Walter Dick and Lou Carey, The Systematic Design of Instruction (Glenview, Ill.: Scott, Foresman, 1985); Jerrold E. Kemp, The Instructional Design Process (New York: Harper, 1985).

2. Keith M. Cottam and Connie V. Dowell, "A Conceptual Planning Method for Developing Bibliographic Instruction Programs," Journal of Academic Librarianship 7:223-28 (Sept. 1981); Patricia A. Kenney and Judith N. McArthur, "Designing and Evaluating a Programmed Library Instruction Text," College \& Research Libraries 45:35-42 (Jan. 1984); Marian I. Miller and Barry D. Bratton, "Instructional Design: Increasing the Effectiveness of Bibliographic Instruction," College \& Research Libraries 49:545-49 (Nov. 1988).

3. Delia Neuman and Rebecca Jackson, MAJIK/1: HyperCard Introduction to the Use of Periodicals. University of Maryland Libraries System. University of Maryland. College Park. Final report to the Council on Library Resources, Cooperative Research Grant Program, CLR Grant \# 4048-F (Washington, DC: June 1990).

4. Members of the Committee included the UMCP Libraries Coordinator for Instructional Services, a faculty member of the College of Library and Information Services with a background in ISD, two UMCP librarians who teach in the Library Instruction Program, and a faculty member of the UMCP English Department representing junior composition teachers whose courses include a library instruction session.

5. Egon G. Guba, "Criteria for Assessing the Trustworthiness of Naturalistic Inquiries," Educational Communication and Technology Journal 29:75-91 (Spring 1981); Egon G. Guba, "Naturalistic Evaluation," New Directions for Program Evaluation 34:23-44 (Summer 1987); Yvonna S. Lincoln and Egon G. Guba, Naturalistic Inquiry (Beverly Hills, Calif.: Sage, 1985), passim; Delia Neuman, "Naturalistic Inquiry and Computer-Based Instruction: Rationale, Procedures, and Potential," Educational Technology Research and Development 37:39-51 (Fall 1989).

6. Kemp, The Instructional Design Process, p. 229. 


\section{APPENDIX A. MAJIK/1 GOALS AND OBJECTIVES}

\section{Introduction to periodical indexes}

GOAL: The user will describe the nature and purposes of indexeS.

OBJECTIVES:

1. The user will define a periodical index and name two different types of periodical indexes.

2. The user will differentiate between a general and a specialized index.

3 . The user will state the titles of three different periodical indexes.

II. Instruction in the use of periodical indexes

GOAL: The user will select appropriate indexes and subject headings and identify the elements of a citation in a basic index.

OBJECTIVES:

1. Given a particular topic, the user will select an appropriate general index for that topic and will locate appropriate subject heading(s) within the index for that topic.

2. The user will identify all the elements in a given citation: article author, article title, periodical title, volume, date, pages.

III. The user will match abbreviated journal titles to the full titles.

GOAL: The user will use subject headings, subheadings, citations, and cross references in index entries.

OBJECTIVES:

1. Given a page from an index, the user will identify the subject heading, a subheading, a citation, and a cross reference.

2. The user will follow a cross reference to its referent.

IV. Instruction in the use of the UMCP Serials List

GOAL: The user will identify locations and holdings for any periodical within the UMCP Libraries.

OBJECTIVES:

1. Given a periodical index citation, the user will identify the UMCP Serials List as a source of information to locate that article.

2. Given a periodical title, the user will select the correct microfiche card to be used and will use the index at the top of the card to locate that title on the card.

3. Given a periodical title, the user will determine the call number for that title.

4. Given a specific issue of a periodical, the user will identify the format of that issue (microfilm, microfiche, bound, unbound) and will determine which library/libraries hold the issue.

V. Instruction in the arrangements of periodicals in the various UMCP Libraries

GOAL: The user will determine where in the Libraries s/he can find a periodical. OBJECTIVES:

1. The user will explain the difference in the ways periodicals are stored in the two largest UMCP Libraries.

2. The user will identify the appropriate strategy for finding periodicals in the other UMCP Libraries.. 